\title{
EL PROBLEMA DE LA ESCALA DE PLANCK
}

\author{
Oscar Monroy Cárdenas ${ }^{\mathrm{a}^{*}}$, Mateo Márquez Jacome ${ }^{\mathrm{a}}$ \\ ${ }^{a}$ Facultad de Ciencias Físicas, Universidad Nacional Mayor de San Marcos Ap. Postal 14-0149, Lima 14, Perú.
}

(Recibido Mayo 01, 2009; Aceptado 29, Julio 2009)

\begin{abstract}
Resumen
Como resultado de investigar el régimen de Planck por medio del propio principio de Planck, el principio de incertidumbre de Heisenberg y la ecuación de existencia de Einstein surge el concepto del punto físico el cual sería una abertura del universo de extensión $10^{-35} \mathrm{~m}$ a través de la cual se produciría $10^{-8} \mathrm{~kg}^{\mathrm{de}}$ materia/antimateria cada $10^{-43} \mathrm{~s}$. Se deduce que ninguna entidad física podría vibrar con una frecuencia superior a $10^{43} \mathrm{~Hz}$. También se deduce que en el régimen de Planck la temperatura sería del orden de $3 \times 10^{32} \mathrm{~K}$. Considerando que las pulsaciones de todas las estructuras físicas provienen del régimen de Planck se deduce que el universo tendría carácter dual. Esto podría cambiar nuestra concepción de espacio como una superficie tridimensional de dos caras.
\end{abstract}

PACS: 75.10.Jm

Palabras claves: Régimen de Planck, Principio de Planck, Principio de incertidumbre de Heisenberg, Ecuación de existencia de Einstein, Punto físico, Extensión del Universo, materia/antimateria.

\begin{abstract}
Searching Planck's Regimen utilizing Planck's Principle, Heisenberg's uncertainly Principle and Einstein's existence equation, it emerges concept of physical point which that would be a hole as extension of $10^{-35} \mathrm{~m}$ through the Universe, so it would produce $10^{-8} \mathrm{~kg}$ of materia/antimateria one a $10^{-43} \mathrm{~s}$. It deduces that any physiscal entity could vibrate at frequencies larger to $10^{43} \mathrm{~Hz}$. Also it deduces, at Planck's Regimen, that temperature would be about $3 \times 10^{32} \mathrm{~K}$ order. Considerating pulsations at Planck's Regimen of each physical structures, it deduces that Universe has dual character. It means that conception about of space, it would be actually a tridimensional surface of two faces.
\end{abstract}

Keywords: Planck's Regimen, Planck's Principle, Heisenberg's uncertainly Principle, Einstein's existence equation, Physical point, Universe extension, matter/antimatter.

\section{Introducción}

La escala de Planck fue introducida con el propósito de explorar posibles dimensiones ocultas donde las fuerzas fundamentales de la Física se unifiquen en una sola superfuerza la cual sería la generadora de todas las fuerzas y todas las estructuras físicas que observamos.

En la Física Cuántica se asocia una escala de energía (o su equivalente, la masa) con una escala de longitud. Se observa que para sondear dimensiones cada vez más pequeñas se requerirán cantidades de energía cada vez mayores. Por ejemplo, para explorar el interior de un protón se requiere disponer de energías equivalentes al menos, 10 veces la masa del protón. Las masas que utilizan las teorías de unificación son de $10^{14}$ veces la masa del protón. Otras teorías sugieren que, para que se manifiesten las dimensiones ocultas, se requiere una cantidad de energía equivalente a $10^{19}$ veces la masa del protón. Esta enorme cantidad se conoce como escala de Planck.

Combinando la constante de gravitación $(G)$, la constante de Planck $(\hbar)$ y la rapidez de la luz en el vacío $(c)$ se obtiene, en la escala de Planck, la unidad de longitud $\left(l_{p} \sim 10^{-35} \mathrm{~m}\right.$, llamada longitud de Planck), la unidad de tiempo $\left(t_{p} \sim 10^{-43} \mathrm{~s}\right.$, llamado tiempo de Planck) y la unidad de masa $\left(m_{p} \sim 10^{-8} \mathrm{~kg}\right.$, llamada masa de Planck). La cantidad $10^{-35} \mathrm{~m}$ se considera la extensión espacial más pequeña descrita con sentido físico. Análogamente, la cantidad $10^{-43} \mathrm{~s}$ se considera el intervalo de tiempo más pequeño que puede describirse con algún significado. Pero la cantidad $10^{-8} \mathrm{~kg}$ es del orden de $10^{19}$ veces la masa del protón y se considera el umbral de energía que se requiere

\footnotetext{
*Correspondig author.e-mail: oscarmonroycardenas@hotmail.com
} 
utilizar para que se manifiesten las posibles dimensiones ocultas donde la Física se describiría con espectacular sencillez.

Uno de los problemas fundamentales de la Física actual es darle significado a estos misteriosos números para justificar la investigación en la Física de altas energías y poder percibir la acción de la superfuerza la cual determinaría la realidad objetiva del universo. Una empresa tan abstracta, como la Física de partículas, constituye el inequívoco testimonio del espíritu materialista del físico tradicional el cual tiene interpretación subjetiva del mundo. Tenemos que darnos cuenta de que la materia es sólo un vehículo a través del cual la naturaleza expresa su lenguaje mediante los fenómenos naturales. ¡La superfuerza que anima la materia no está en la materia! Debe estar en una dimensión extra a las dimensiones que percibimos ¿Cuál será esa dimensión? Entonces ¿saben los físicos tradicionales lo que están haciendo? Si lo saben, entonces ¿hacia dónde va la ciencia? [1].

Nuestra investigación es también a un nivel fundamental. Abordaremos el problema de la escala de Planck desligándonos de los prejuicios de la Física convencional con el propósito de darle una interpretación diferente, no materialista, a la escala de Planck, Lo primero que tenemos que tener en mente es que las constantes cosmológicas $G, \hbar$ y $c$ son números que fueron obtenidos por mediciones realizadas en escenarios inerciales. Pero ¿seguirán siendo constantes en escenarios no inerciales? No olvidemos que la materia es un sistema de referencia no inercial. En realidad, todas nuestras mediciones son realizadas en escenarios acelerados. Por consiguiente, no sería objetivo considerar a $G, \hbar$ y $c$ como constantes absolutas.

Debido a la crisis del colapso gravitatorio [7,8] la mayoría de físicos del mundo tienen la creencia que en el régimen de Planck estaría la explicación de todas las fuerzas que se consideran fundamentales, así como la formación de todas las estructuras físicas. Motivados por esta causa nuestra investigación del régimen de Planck estará basado en el propio principio de Planck, el principio de incertidumbre de Heisenberg y la ecuación de existencia de Einstein. Trataremos de interpretar con la mayor objetividad posible los resultados que obtengamos para poder contribuir con observaciones útiles al progreso de la Física.

\section{Interpretación del régimen de Planck}

En las teorías físicas actuales [2,3] se intentan unificar los campos gravitatorio, electromagnético y nuclear en un solo campo, el cual permita describir progresivamente la realidad objetiva. Se plantea que las menores longitudes de las microestructuras físicas tienen como límite inferior a la longitud de Planck

$l_{P}=\left(\frac{G \hbar}{c^{3}}\right)^{1 / 2} \sim 10^{-35} \mathrm{~m}$

Aquí $G, \hbar$ y $c$ son las constantes fundamentales de la naturaleza: constante de gravitación, constante de Planck y la rapidez de la luz en el vacío respectivamente.

A esta escala de longitudes tan extraordinariamente pequeña, con respecto a la escala humana, los efectos cuánticos y gravitatorios se consideran importantes. Además, es razonable suponer que existen procesos de creación de materia y antimateria. Por consiguiente, debemos tener en cuenta la energía de existencia de Einstein:

$E_{o}=m_{o} c^{2}$,

donde $m_{o}$ es la masa en reposo de la partícula, conjuntamente con la ecuación cuántica de Planck:

$E=\frac{h c}{\lambda}$,

donde $\lambda$ es la longitud de onda asociada al fotón. Si con la cantidad $E$ se crea una partícula y una antipartícula tal que $E / 2=E_{o}$, entonces $\lambda=\lambda_{\mathrm{C}} / 2$, donde:

$\lambda_{C}=\frac{h}{m_{o} c}$.

Esta cantidad se llama longitud de onda Compton de la partícula. Parece indicar el tamaño aparente o efectivo de una microestructura.

Sin embargo, una interpretación más rigurosa de la Ec.(4), basada en la relación de incertidumbre posición-momentum, consiste en afirmar que es imposible localizar e identificar a una partícula en una región del espacio menor que la longitud de onda Compton [3]. En otras palabras, la longitud de onda Compton es el límite inferior de longitud que debe tener toda microestructura física para poder detectar indirectamente su existencia. Esto se expresa por la condición:

Tamaño de la microestructura $\gtrless \lambda_{C}$

Por ejemplo, para que exista el núcleo atómico, su tamaño debe ser mayor que la longitud de onda Compton del protón $\left(\gtrless 10^{-15} \mathrm{~m}\right)$.

Se puede dar otro significado importante a la longitud de onda Compton, combinando la energía de 
existencia de Einstein (2) con la relación de incertidumbre energía-tiempo:

$$
\Delta E \Delta t \gtrless h
$$

Pero, sabemos que esta relación implica la posibilidad de suspender el principio de conservación de la energía durante el intervalo de tiempo $\Delta t$, y puede aparecer espontáneamente en el universo la cantidad de energía $\Delta E \sim m_{o} c^{2}$ para crear transitoriamente una partícula, llamada partícula virtual. Según la relación (6), esta clase de partícula existiría durante un intervalo de tiempo $\Delta t$ tal que:

$$
\Delta \mathrm{t} \gtrless \frac{h}{m_{o} c^{2}}
$$

Luego, al insertar la Ec.(4) en (7) se deduce la condición:

$\lambda_{C} \leqslant c \Delta t$

Por tanto, la longitud de onda Compton significa el máximo alcance que puede experimentar una partícula virtual en una región del espacio libre antes que desaparezca de nuestro mundo [4].

Análogamente, ¿qué significado tendría la frecuencia Compton? De la relación (7) es claro que $1 / \Delta t \leqslant m_{o} c^{2} / h$. Si $\Delta t$ se identifica como el periodo de la onda asociada a la partícula virtual, entonces, se obtiene la frecuencia Compton:

$v_{C} \cong \frac{m_{o} c^{2}}{h}$

Este resultado indica la máxima frecuencia de vibración de una partícula virtual que puede ser inducida por una fuerza para que su existencia tenga efectos medibles. El caso $m_{o}=0, v_{C}=0$ carece de sentido. El caso de especial significado emerge cuando en la Ec.(9) se reemplaza $m_{o}$ por la masa de Planck $m_{P}$, la cual se deduce de la igualdad $\lambda_{C}=l_{P} \mathrm{y}$ de las expresiones (1) y (4), siendo el resultado:

$m_{P}=\left(\frac{\hbar c}{G}\right)^{1 / 2} \sim 10^{-8} \mathrm{~kg}$

La introducción de la masa de Planck en la Ec.(9) conduce al siguiente resultado:

$$
\omega^{*}=2 \pi v^{*} \cong\left(\frac{c^{5}}{G \hbar}\right)^{1 / 2}
$$

Esta cantidad es una frecuencia fundamental intrínseca a la escala de Planck. Está determinada por las tres constantes cosmológicas: $c=3 \times 10^{8} \mathrm{~m} / \mathrm{s}, G=$ $6,67 \times 10^{-11} \mathrm{Nm}^{2} / \mathrm{kg}^{2}$ y $\hbar=1,05 \times 10^{-34} \mathrm{Js} . \quad \mathrm{Al}$ reemplazar estos valores en la fórmula (11) se obtiene la frecuencia natural:

$$
v^{*} \sim 10^{43} \text { vibraciones / segundo }
$$

Nótese que esta frecuencia es del orden de $10^{29}$ veces la frecuencia de la luz visible, es decir:

$v^{*} \sim 10^{29} v_{l u z}$

Esta enorme frecuencia (respecto a la frecuencia de la luz visible) correspondería a las pulsaciones de una entidad física universal. Ninguna entidad física podría vibrar con una frecuencia mayor o igual que la cantidad $v^{*}$.

Ahora investiguemos con qué rapidez se movería una partícula en el régimen de Planck. Mediante la relación de incertidumbre posición-momentum se tiene:

$\Delta x \Delta p \gtrless h$

Si $\Delta x \sim 10^{-35} m$ y como $h \sim 10^{-34} \mathrm{Js}$, entonces se obtiene $\Delta p \gtrless 10 \mathrm{~kg} \mathrm{~m} / \mathrm{s}$, o también $m_{P} v \gtrless 10 \mathrm{kgm} / \mathrm{s}$, por consiguiente se deduce que:

$v \gtrless 10^{9} \mathrm{~m} / \mathrm{s}>c$

Este resultado conduce a la conclusión de que en el régimen de Planck las partículas virtuales se moverían más rápido que la luz; su energía es tan grande que a esta escala sólo tendría sentido pensar en la existencia de una fuerza impulsiva extraordinaria (superfuerza) la cual no sólo sería responsable de la transmisión de energía ultraalta, sino también de un nuevo fenómeno físico de creación de materia y antimateria.

Del mismo modo que la rapidez de la luz en el vacío representa un límite superior para la rapidez de traslación de los cuerpos materiales, la frecuencia fundamental $v^{*}\left(\right.$ o $\left.\omega^{*}\right)$, representaría un límite superior para la frecuencia de vibración de todas las entidades físicas. Por tanto, es razonable proponer el siguiente postulado:

Ninguna entidad física puede vibrar con una frecuencia mayor o igual que la frecuencia fundamental $v^{*}=10^{43}$ vibraciones/segundo

Con la suposición de que el universo observable existe a partir de la escala de Planck, le llamaremos a esta conclusión postulado de existencia y a la frecuencia fundamental $v^{*}$ le llamaremos frecuencia de existencia. 


\section{Discusión}

La implicación inmediata del postulado de existencia es que en la escala de Planck se tendría la máxima rapidez con que podría radiar una entidad física, y que a medida que aumenta la escala, la frecuencia de vibración de las microestructuras va disminuyendo. Lo intrigante es saber qué mecanismo existe en el espacio para que la frecuencia de vibración de las microestructuras vaya disminuyendo progresivamente tal como se observa en las subsiguientes microestructuras: núcleos $\left(\sim 10^{14}\right.$ vibraciones/segundo $), \quad$ átomos $\quad\left(\sim 10^{8}\right.$ vibraciones/segundo $)$ y moléculas $\quad\left(\sim 10^{4}\right.$ vibraciones/segundo), como se muestra en el esquema de niveles de vibración mostrado en la Fig.1. Esto se podría extrapolar llegando a las macroestructuras, como nosotros, los seres humanos $(\sim 1$ vibración/s), que emitimos radiación infrarroja, y a todo el resto del universo a gran escala. Además, esto explicaría, que el universo a gran escala nos parece estático debido a que la frecuencia de vibración de las estrellas y nebulosas es mucho menor que $1 \mathrm{~Hz}$.

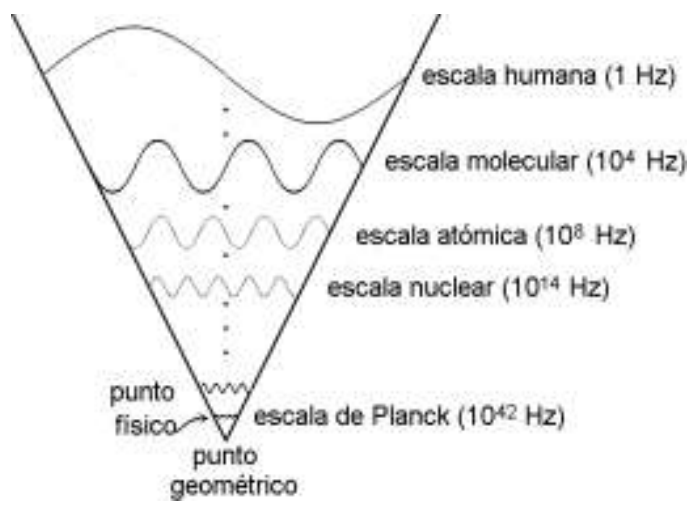

Fig. 1. Frecuencias características de vibración de las estructuras físicas desde la escala de Planck.

Notemos que cada escala de estructuras está caracterizada por un intervalo de frecuencias de vibración donde existirá una tendencia al equilibrio térmico. Por ejemplo, en la escala humana habrá una tendencia al equilibrio térmico en el intervalo de frecuencias que lo caracteriza (que es del orden de 1 $\mathrm{Hz}$ ). A estos niveles de energía le podemos llamar estados estacionarios o pulsaciones del tiempo porque corresponden a jerarquías de estructuras estables que evolucionan en el tiempo.

Por otro lado, el resultado (14) nos dice que en la escala de Planck una partícula virtual con la masa de Planck (del orden de $10^{-2}$ miligramo) podría adquirir una rapidez del orden de $10 c$, donde $c=3 \times 10^{8} \mathrm{~m} / \mathrm{s}$. Según el postulado de existencia, ésta rapidez podría ser inducida por una influencia externa al universo (superfuerza) cuyas pulsaciones son del orden de $10^{43}$ $\mathrm{Hz}$.

Si la masa de la partícula virtual en la escala de Planck fuese del orden de $10^{-13} \mathrm{~kg}$ adquiriría una rapidez de $10^{6} \mathrm{c}$; si su masa fuese del orden de $10^{-19}$ $\mathrm{kg}$ su rapidez sería $10^{12} \mathrm{c}$, y si la masa fuese del orden de $10^{-31} \mathrm{~kg}$ (comparable a la de un electrón) su rapidez sería $10^{24} \mathrm{c}$. En consecuencia, en la escala de Planck las ecuaciones de la relatividad especial de Einstein no son válidas. La energía de las partículas virtuales es tan grande que sólo tiene sentido pensar en un nuevo fenómeno físico de creación de materia y antimateria, o mejor dicho es necesario ¡adoptar un nuevo concepto de materia y antimateria, así como de la luz!

La relación de incertidumbre energía-tiempo (6) plantea la posibilidad de que el universo sea un sistema abierto (durante un intervalo de tiempo) para que ingrese energía. A su vez, la ecuación de Einstein (2) plantea la posibilidad de que con la energía (que ingresa al universo) haya creación de materia y antimateria. Entonces tendría que existir una abertura a través de la cual procederían las partículas virtuales en la forma de pares partícula/antipartícula. Esta abertura tendría una extensión de $10^{-35} \mathrm{~m}$ y le llamaremos punto físico (a diferencia del punto geométrico que se define como un segmento de longitud cero). Según el resultado (14), en el régimen de Planck, las partículas virtuales fluirían con rapidez $v>c$ (ver Fig. 2).

El postulado de existencia sugiere simular al punto físico como si fuese un oscilador armónico el cual sería inducido a vibrar por una acción externa al universo cuya frecuencia es del orden de $10^{43} \mathrm{~Hz}$. Esta enorme frecuencia significa que en el régimen de Planck la temperatura es muy alta. En efecto, si las fluctuaciones térmicas son comparables a las fluctuaciones cuánticas, la temperatura sería:

$T \sim \frac{h v^{*}}{2 \kappa_{B}} \sim 3 \times 10^{32} \mathrm{~K}$

Aquí, $\kappa_{B}=1,38 \times 10^{-23} \mathrm{~J} / \mathrm{K}$ es la constante de Boltzmann (el mismo resultado (15) se obtiene usando la ley del desplazamiento de Wien). Por consiguiente, el punto físico se comportaría como un cuerpo negro absorbiendo y emitiendo radiación térmica [6]. Pero, ¿qué hay en el régimen de Planck? $\mathrm{Si}$ el punto físico se comporta como un cuerpo negro, entonces lo que habría sería ¡radiación condensada en estado de equilibrio termodinámico!

Por consiguiente, es necesario definir el concepto de condensación de la radiación. Para que estas ideas no queden confusas, imprecisas e insatisfactorias, se requiere disponer de un nuevo principio físico sobre la propagación de los rayos luminosos en un 
escenario ultraacelerado, como lo es el régimen de Planck, con el propósito de definir a partir de esta escala la unidad de distribución espectral de energía del cuerpo negro con la que identificaríamos a los sistemas naturales. ¿Estaríamos hechos de estos cuantos de energía provenientes de una influencia externa al universo?

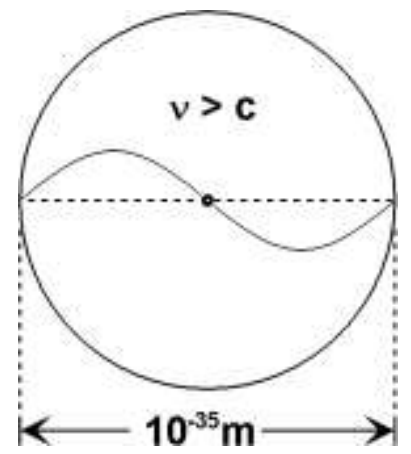

Fig. 2. En el régimen de Planck cualquier entidad física fluiría con una rapidez superior a la rapidez de la luz en el vacío.

Imaginemos que una influencia externa al universo actúa como una válvula inyectando orden en una revitalización continua de energía, abriéndose y cerrándose según el ritmo de la pulsación. Este mecanismo puede ser representado (en el régimen de Planck) por pulsos armónicos de duración $t_{P}=10^{-}$ ${ }^{43} \mathrm{~s}$, como sigue:

$$
f(t)=a(t) \cos \omega^{*} t
$$

donde:

$$
a(t)= \begin{cases}1, & \text { para }|t|<t_{P} \\ 0, & \text { para }|t|>t_{P}\end{cases}
$$

Utilizando el análisis de Fourier, el espectro vibracional del pulso $\mathrm{f}(\mathrm{t})$ está descrito por:

$$
F(\omega)=\frac{\operatorname{sen}(\omega-\omega *) t_{P} / 2}{\omega-\omega *}+\frac{\operatorname{sen}(\omega+\omega *) t_{P} / 2}{\omega+\omega *}
$$

En la Fig.3 se muestra la gráfica de la amplitud espectral hipotética $F(\omega)$ en función de la frecuencia $\omega$ de la influencia externa. Obsérvese que existen dos distribuciones simétricas de energía, y por consiguiente, dos frecuencias fundamentales simétricas $\omega^{*}$ y $-\omega^{*}$. Una de las distribuciones de energía puede interpretarse como la imagen especular de la otra. Esto nos induce a postular que el universo tiene simetría de reflexión. Si el espacio es considerado como una superficie tridimensional, entonces tendría carácter dual, es decir ¡tiene dos caras!

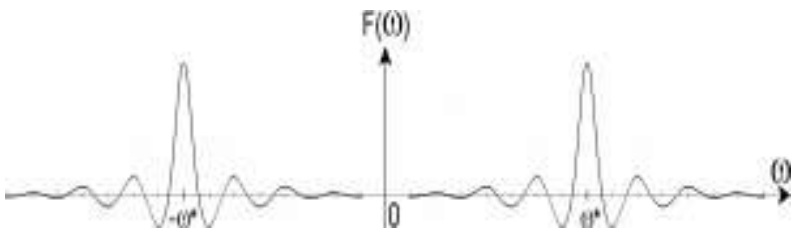

Fig. 3. Espectro vibracional hipotético que se obtendría si el punto físico tuviera pulsaciones armónicas. Éstas distribuciones de energía simétricas sugieren que el universo tendría carácter dual

El primer término en la Ec.(18) significaría emisión de radiación (con frecuencia $\omega^{*}$ ) y el segundo término significaría absorción de la radiación (con frecuencia $-\omega^{*}$ ). La radiación suministrada por la influencia externa al universo sería absorbida por una cara del espacio y emitida por la otra cara del espacio.

Debido a la simetría especular, intrínseca al universo, una observación interesante es que la energía total del universo sería cero. Por ejemplo, si a la frecuencia de existencia $\omega^{*}$ le corresponde una energía de existencia $m_{o} c^{2}$, entonces a la frecuencia $\omega^{*}$ le correspondería la energía $-m_{o} c^{2}$, y por consiguiente, la energía total es cero, ipero no es cero en cada cara del espacio! La superfuerza externa al universo inyectaría la cantidad de energía $\Delta E=$ $2 m_{o} c^{2}$, según el ritmo de la pulsación a partir de la escala de Planck. Estas cantidades de energía se transmitirían a todo el universo canalizándose de acuerdo a una ley general de propagación de los rayos luminosos en marcos no inerciales por descubrir.

Parece paradójico considerar al punto físico como fuente y sumidero a la vez de radiación. Pero si pensamos que la radiación procede de una dimensión extra a nuestro espacio tridimensional dual (que llamaremos dimensión del tiempo o simplemente el tiempo) entonces esta proposición podría ser aceptable. Si imaginamos que todos los posibles caminos perpendiculares al espacio (superficie tridimensional de dos caras) conducen al punto físico, entonces nos parecería que el punto físico estuviera en todas partes y sería como una entrada a la dimensión del tiempo.

Sólo si nuestra mente está en el tiempo, podríamos percibir el universo de la forma que es (uniforme, homogéneo e isotrópico a gran escala; ¡lo mismo sería válido a muy pequeña escala!). Entonces nuestra mente estaría en un sistema de referencia privilegiado: el tiempo. ¿Podríamos concebir a la dimensión del tiempo como un sistema de referencia inercial absoluto respecto al cual se describa la realidad objetiva? 
Si todos los objetos materiales son afectados por la corriente del tiempo, entonces, ¿por qué no podemos pensar que el tiempo es una influencia externa al universo, es decir, la superfuerza? Las pulsaciones intrínsecas que detectamos en los sistemas naturales nos sugieren que la acción del tiempo está en todas partes. ¿Somos el pulso del tiempo?

Las vibraciones del punto físico debido a una influencia externa al universo (la acción del tiempo) implican fluctuaciones de la escala de Planck. La emisión de radiación iría acompañada de una expansión del punto físico a la longitud $\mathrm{I}_{\mathrm{p}}+\Delta \mathrm{r}, \mathrm{y}$ por el contrario la absorción iría acompañada de una compresión del punto físico hasta la longitud $\mathrm{I}_{\mathrm{p}}-\Delta \mathrm{r}$, siendo $\Delta \mathrm{r}$ la variación de la longitud de Planck. Estas fluctuaciones (modos vibracionales) constituirían el origen de nuestra noción de carga eléctrica.

Si el punto físico se comporta como un cuerpo negro, entonces ipodríamos pensar que tiene las propiedades de una estrella! Pero en un universo abierto el Sol y las estrellas ya no serían cuerpos finitos, sino cavidades resonantes infinitamente profundas (es decir, aberturas hacia la dimensión del tiempo) Por consiguiente, se descartaría la hipótesis de que el Sol y las estrellas se alimenten de la energía disipada de las reacciones nucleares. En otras palabras, la luz, el calor, el hidrogeno, el helio, el carbono, el nitrógeno, el oxígeno y todos los demás elementos químicos serían creados por un mecanismo que aún desconocemos. Por tanto, el Sol y las estrellas no serían simples fuentes de consumición de hidrógeno. Pensando en núcleos atómicos y procesos nucleares ¡nunca llegaremos a adorar al Sol!

\section{Conclusiones}

La interpretación de la escala de Planck por medio del principio de Planck, el principio de incertidumbre de Heisenberg y la ecuación de la energía de existencia de Einstein conduce a la idea del punto físico, el cual se define como una abertura del universo de extensión $10^{-35} \mathrm{~m}$ a través de la cual se produce $10^{-8} \mathrm{~kg}$ de materia/antimateria cada $10^{-43}$ $s$.

Ninguna entidad física puede vibrar con una frecuencia superior a la frecuencia del punto físico: $10^{43} \mathrm{~Hz}$.

El régimen de Planck sería una cavidad resonante donde existiría un patrón de flujo (ultraacelerado) de radiación condensada rotante $y$ vibrante cuya temperatura es del orden de $3 \times 10^{32} \mathrm{~K}$. El patrón de flujo de radiación estaría descrito por una ley general de propagación de los rayos luminosos en marcos inerciales.

Si las pulsaciones que percibimos en los sistemas naturales provienen de una influencia externa al universo observable, se deduce por medio de su espectro vibracional que el universo tendría carácter dual con frecuencias de existencia simétrica $\omega^{*}$ y $\omega^{*}$. Esto sugiere considerar el espacio como una superficie tridimensional de dos caras. Por consiguiente, si cada $10^{-43} \mathrm{~s}$ ingresa al universo una cantidad de energía $\Delta E$ ésta se desdoblará en una cantidad positiva y otra cantidad negativa, ambas de igual magnitud, siendo la energía total igual a cero. Esto significaría que una cara del espacio se comportaría como un absorbente perfecto y la otra como un emisor perfecto.

Si el punto físico se comporta como una cavidad resonante en equilibrio termodinámico (cuerpo negro) entonces podría ser identificado como la verdadera esencia de una estrella. Por tanto, en la escala de Planck el punto físico nos proporcionaría la unidad de distribución espectral de energía del cuerpo negro con la que definiríamos el estado de un sistema natural, así como su verdadera identidad. Por tanto, podríamos responder las preguntas: ¿Quiénes somos? ¿de qué estamos hechos?

\section{References}

[1]. Max Planck. ¿Adónde va la Ciencia? Ed. Losada S.A., Buenos Aires. 1941.

[2]. Brian Greene. El Universo Elegante. Crítica Planeta. Barcelona 2006.

[3]. Stephan Hawking. El Universo es una Cáscara de Nuez. Crítica Planeta. Barcelona 2003.

[4]. P. Davies. El Universo Accidental. Biblioteca Científica Salvat (versión original publicada por Cambridge University), 1986.

[5]. Steven Weinberg. Gravitation and Cosmology: Principles and Applications of the General Theory of Relativity. John Wiley \& Sons, Inc. New York, 1972.

[6]. Louis De Broglie. La Física Nueva y los Cuantos. Guest Editor: Dennis Dicks (2001).

[7]. J.A.Wheeler, C.W.Misner y K.Thorne. Gravitation. Freeman 1973

[8]. J.A. Wheeler. Un Viaje por la Gravedad y el Espacio-Tiempo. Alianza Editorial S. A. 1994.

[9]. Edited by Jeremy Bernstein and Gerald Feinberg. Cosmological Constants. Papers in Modern Cosmology, New York, 1986. 